\title{
An elastic pulsed-jet thruster for Soft Unmanned Underwater Vehicles
}

\author{
Francesco Giorgio Serchi, Andrea Arienti, Ilaria Baldoli and Cecilia Laschi, Senior Member, IEEE
}

\begin{abstract}
This paper reports on the development of a new kind of unmanned underwater vehicle which draws inspiration from cephalopods both in terms of morphology and swimming routine. The robot developed here is the first in its kind, being a soft aquatic robot which travels in water by pulsed-jet propulsion. The general design principles of this innovative kind of underwater robot are illustrated and a first prototype is built and tested. The experiments demonstrate an inverse correlation between the frequency of pulsation and the speed of the robot. A mathematical model which associates the kinematics of the pulsating routine to the dynamics of the swimming is devised and compared with the experiments in order to better investigate the interplay of the various design parameters.
\end{abstract}

\section{INTRODUCTION}

Along with the ever growing interest for oceans and marine technologies, the need comes to perform construction, maintenance and surveying tasks in always more challenging scenarios. Autonomous Underwater Vehicles (AUVs) and Remotely Operated Vehicles (ROVs) are being looked upon as the indispensable tool to deal with the increasing spectrum of applications which marine technologies rely on. In particular, ROVs have demonstrated their fitness at dealing with complex tasks in extreme conditions. Nonetheless, new breeds of robots are ready to overtake the traditional Unmanned Underwater Vehicles (UUVs) by demonstrating their skills for a whole new set of applications so far hardly imaginable for standard underwater robots.

In recent times, a very fascinating trend of research is to look at nature as a source of inspiration for the design of new robots with unprecedented skills. Underwater robotics has greatly benefited from this approach and a broad range of innovative robots have appeared which are capable of amazing feats, well beyond what is observed in standard, commercially available ROVs. Robotic fish (e.g. [1], [2], [3]) now manifest their aptitude for fast-start manoeuvres, promising to provide an exceptional asset over common propeller-driven robots in highly perturbed, wave or current dominated environments. Snake like robots demonstrate their proneness at working in constrained, cramped, unstructured environments and hexapod or crab-like robots suggest that operations over the sea bottom or underneath the hull of a ship could soon enough be regarded as standard procedures.

At present, several aquatic mode of locomotions have been replicated by bioinspired robots. However, the pulsedjet swimming of cephalopods has so far gained only limited

This work was supported by the European Commission in the frame of the FP7 CFD-OctoProp ERG and the OCTOPUS IP.

All authors are with the Centre on Sea Technology and Marine Robotics, the Biorobotics Institute, Scuola Superiore Sant'Anna, Pisa, Italy, email: f.serchi@sssup.it recognition (i.e. [4], [5], [6], [7], [8]) and never in the context of soft robotics. This is somewhat surprising given that this mode of swimming has two major merits: first, pulsedjetting is known to provide more thrust than the continuous outflow case, due to vortex ring formation at the nozzleexit; in addition, this mode of locomotion has an efficiency comparable to that of fish but does not require an underlying rigid structure. The implication of this is that by taking inspiration from cephalopods it will be possible to design highly manoeuvrable, underwater soft robots.

Soft robotics (see [9], [10], [11], [12], [13], [14]) is a fairly new trend which attempts to design robots with high intrinsic compliance and hyper-redundancy. The benefits provided by such properties lie in the reduced risk of causing harm, as well as damaging the surrounding environment and the robot itself. While terrestrial environments pose a significant hindrance to soft robots, because the lack of rigid structures forces them to travel by crawling, serpentine or worm-like locomotion, in water this major drawback is vanquished. Cephalopoda are the living proof that, in the aquatic environment, the lack of a skeletal supportive structure does not prevent from sporting outstanding swimming skills and manipulation capabilities. Indeed, soft manipulators are being developed which take inspiration from the octopus arm [15], [16], [17]. It is the authors opinion that the aquatic environment and the spectrum of engineering applications which are performed there is where the asset of soft robots could be best employed. The reduced risk of damage, the capability to adjust through cramped spaces and narrow apertures, the reduced weight and the intrinsic safety for the human operator are only a few examples where the advantage of soft robots could be achieved in marine applications.

In this paper the focus is put on the development and modelling of a soft underwater robot whose general actuation principle and swimming routine are based on a functional approximation of those observed in cephalopods. Given the novelty of the prototype presented here, we will be referring to this new kind of robot as Soft Unmanned Underwater Vehicle, or SUUV.

\section{DESIGN PRINCIPLE}

Pulsed-jet propulsion works by repetitive cycles of ingestion of ambient water in a chamber and subsequent compression of such chamber. The abrupt pressurization of the volume stored inside the chamber drives the acceleration of fluid across a nozzle. The reaction generated by the expulsion of a finite amount of fluid pushes the chamber backward. The expulsion of this slug of fluid occurs along with the formation of a range of ordered or less-ordered 
vortical structures. The importance of such structures in determining a positive feedback onto the thrust generated has been long explored and eventually associated with an optimal vortex formation time for which an over-pressure term arises at the nozzle-exit that drives the onset of additional thrust, [18], [19]. In living animals, the pulsation cycle occurs via the interplay of radial and longitudinal muscles located in the thickness of the mantle wall [20].

In the present work the compression/inflation cycle is performed by means of a simple actuation routine based on a cable driven crank-mechanism. This kind of actuator allows to perform a swimming routine very resemblant to that of swimming cephalopods and at the same time guarantees that only limited rigid structures are essential to the operation of the robot. The generic prototype is composed of an elastic shell which acts as the chamber where ambient fluid is stored before being expelled. The compression of this chamber is driven by a number of inextensible cables fitted, at one end, to the shell wall, and, at the other end, to some mechanism which pulls the cables (and the shell walls attached to them) inwards. The external shell pressurizes the fluid stored in the chamber and in this way accelerates it across an outflow. The cables are cyclically pulled and released. Once they are released, the stresses within the thickness of the shell walls act to bring the walls back in their original, unstrained state. In doing so, the shell inflates and the pressure drop occurring within drives ambient water back into the chamber. Then the cables are pulled again. The repetition of this routine gives rise to the pulsation cycle which permits the robot to swim. The major difference between the robot and its biological counterpart lies in the way the contraction of the shell takes place. In living cephalopods the contraction occurs homogeneously over the shell thickness, hence providing an isotropic compression of the internal volume of fluid. On the other hand, in the robot the compression occurs at those spots where the cables are fitted, hence giving rise to a bucklinglike deformation of the external shell in the proximity of the cable attachment. These are the general concept which enable the development of a soft robot swimming by pulsedjet propulsion. The reader is referred to [21] for a thorough discussion on the choice of actuation criteria and actuator design. Below the design of the prototype employed here is presented.

TABLE I

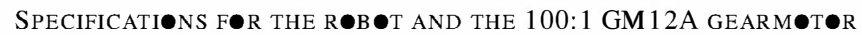

\begin{tabular}{|l|c|}
\hline Total Robot Weight $(\mathrm{g})$ & 550 \\
\hline Max Robot Length $(\mathrm{mm})$ & 210 \\
\hline Max Robot Diameter $(\mathrm{mm})$ & 80 \\
\hline Average Density $\left(\mathrm{g} / \mathrm{cm}^{3}\right)$ & 1.1 \\
\hline Max Cable Contraction $(\mathrm{mm})$ & 28 \\
\hline Max Expelled Volume $(\mathrm{ml})$ & 100 \\
\hline Motor Unloaded Speed $(\mathrm{rpm})$ & $85(3 \mathrm{~V})-169(6 \mathrm{~V})$ \\
\hline Motor Max Torque $(\mathrm{N} \cdot \mathrm{m})$ & $0.110(3 \mathrm{~V})-0.192(6 \mathrm{~V})$ \\
\hline
\end{tabular}

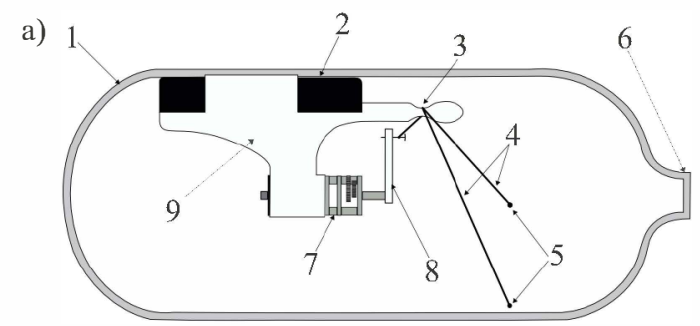

b)

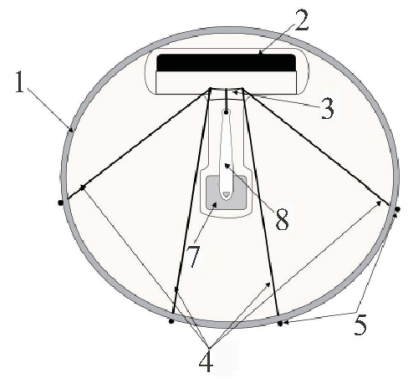

Fig. 1. Schematic depiction of the lateral (a) and frontal (b) view of the robot after assemblage. The numbers respectively refer to: (1) the silicone shell, (2) the batteries, (3) the fixed pulley, or fairlead, (4) the cables, (5) cable attachments, (6) outflow nozzle, (7) the gearmotor, (8) the rod, (9) the PCL mould.

\section{ROBOT CONSTRUCTION}

The robot is composed of an external torpedo-shaped shell of $5.0 \mathrm{~mm}$ thick, EcoFlex ${ }^{\mathrm{TM}} 00-30$ silicone, Fig. 1 and 2 . The torpedo is, in fact, composed by a hollow cylinder with two hollow semi-spheres at its ends. One of the semi-sphere has an outlet cut in, which can host interchangeable nozzles. Another crescent-shaped aperture is carved into the main cylindrical body of the robot; this acts as a selective inflow valve which gets sealed during the expulsion phase and lifts during inflation, enabling the inflow of ambient fluid and in this way the refill of the chamber, see [21]. The actuator is lodged in the center of the cylindrical chamber. This is composed of a mould of Polycaprolactone (PCL) which includes a pair of Lithium Polymer Batteries (3.7 V, 3000 mAh), a GM12A Solarbotics gearmotor (see Table I) and a fixed pulley, as depicted in Fig.1. Insulation of the electronics is guaranteed by immersion in the silicone layer and further sealing with Sil-Poxy ${ }^{\mathrm{TM}}$ silicone adhesive, while the gearbox of the motor is fitted in a grease filled waterproof case. A $14.0 \mathrm{~mm}$ long rod is fitted onto the shaft of the gearmotor and the cables are fixed to this rod. Thank to the rotation of the rod, the cables are cyclically pulled and released. The cables run across the fixed pulley and then are connected to distinct points along a cross section of the silicone shell, see Fig.1. The employment of the fixed pulley, or fairlead, as it would be referred to in the nautical context, allows all the cables to be pulled from the same spot and to the same extent, regardless of their position of attachment over the silicone shell. In this prototype, 4 nylon cables have been used, fitted at equispaced points over one single cross section of the shell.

The PCL mould is glued to the main silicone body in order to hold the actuator in place during activation. The voltage 


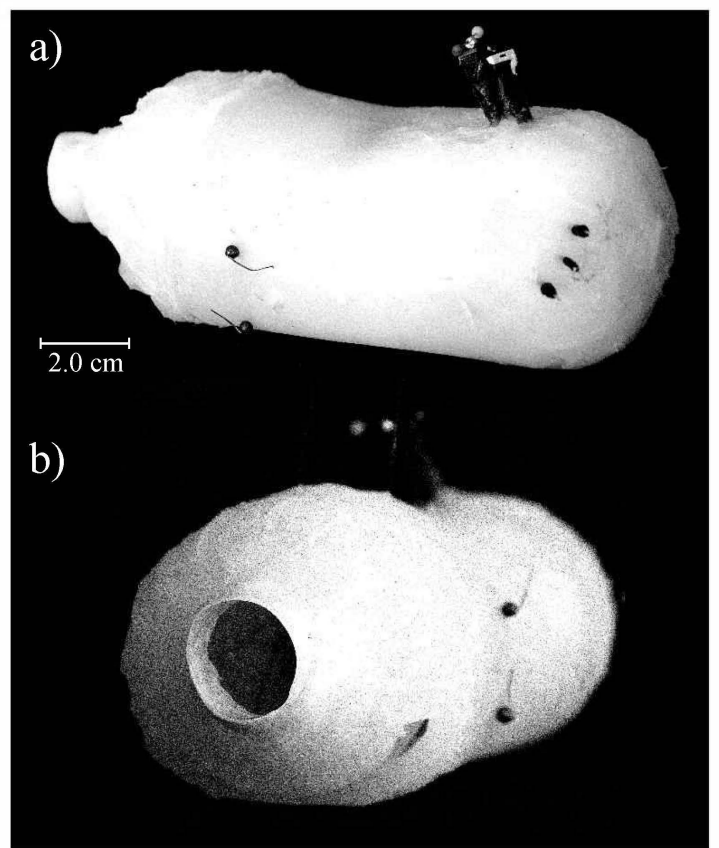

Fig. 2. Rear (a), and frontal (b) view of the robot.

across the motor is adjusted via a potentiometer, which, in turns, provides a way to vary the frequency of pulsation of the elastic chamber. In its final stage, the robot is $210.0 \mathrm{~mm}$ long from the foremost to the rearmost tip and has a $40.0 \mathrm{~mm}$ radius; it weights $550 \mathrm{~g}$ and has an average density of 1.1 $\mathrm{g} / \mathrm{cm}^{3}$, making it slightly negatively buoyant. The silicone constitutes $97 \%$ in mass of the whole robot. The maximum estimated expellable volume is $100 \mathrm{ml}$.

\section{ROBOT TEST}

The robot velocity along straight paths underwater is tested in order to assess its performances as a function of frequency of pulsation and nozzle geometry. These parameters are related not only to the amount of fluid expelled, but also to the ordered vortex structure which are known to positively affect the production of thrust. Three different cylindrical nozzles are tested, which we will refer to as Nozzle-1 (length: $20.0 \mathrm{~mm}$; diameter: $30.0 \mathrm{~mm}$ ), Nozzle-2 (length: $60.0 \mathrm{~mm}$; diameter: $30.0 \mathrm{~mm}$ ) and Nozzle-3 (length: 60.0 $\mathrm{mm}$; diameter: $20.0 \mathrm{~mm}$ ). Each of these are tested at 1.88 $\mathrm{Hz}$ and $2.66 \mathrm{~Hz}$ pulsation frequencies, respectively achieved at about $8 \mathrm{~V}$ and $6.5 \mathrm{~V}$. The tests, listed in Table II, were performed in a $1150 \mathrm{~mm}$ long, $590 \mathrm{~mm}$ wide and $500 \mathrm{~mm}$ deep tank. The tests are recorded with a digital camera at 25 fps and subsequently treated with an image tracking software and a Savitzky-Golay low-pass filter to process the displacement and velocity temporal profiles. Because the robot is slightly negatively buoyant, a small float is linked to it, allowing the robot to travel below the free surface. The float slides along a wire held at the ends of the tank in order to help the robot maintain a straight path during the testing. Despite the float being shaped so to minimize drag at the free surface, a number of errors arise from the present setup. The performance of the robot is partially hindered by
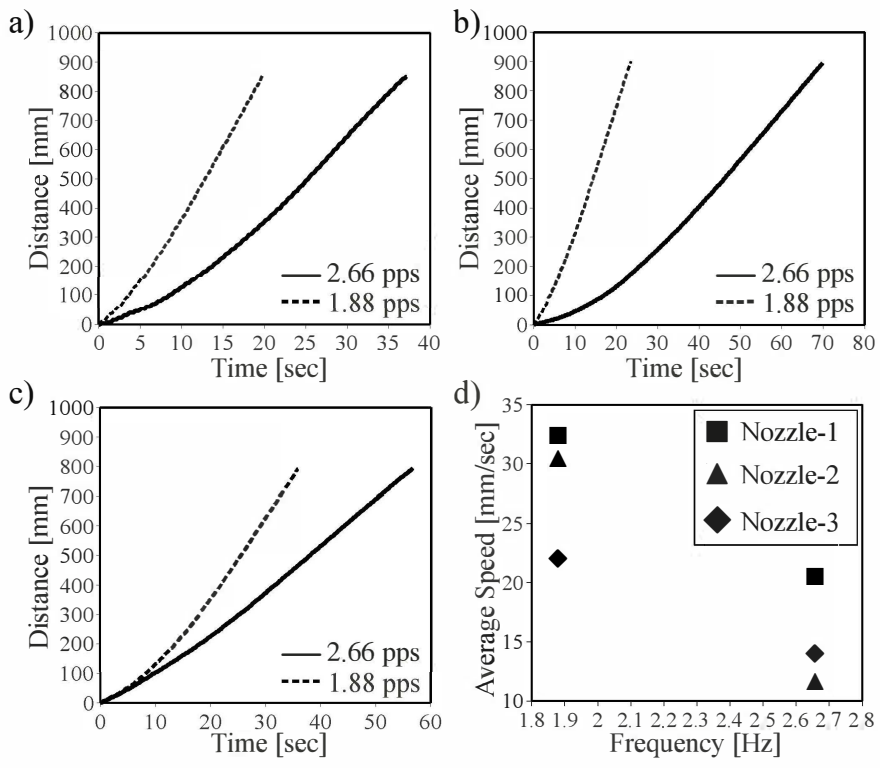

Fig. 3. Temporal evolution of the displacement of the robot for Nozzle-1 (a), Nozzle-2 (b) and Nozzle-3; in (c) the average speed for each nozzle is plotted against the pulsation frequency.

the drag, wave-induced drag and added mass of the float. In addition, the waves generated by the oscillations of the float push the robot backwards as it approaches the far end of the tank.

The results from the tests are briefly illustrated in Fig. 3. Subfigures (a), (b) and (c) of Fig. 3 respectively represent the temporal displacement of the robot for Nozzle-1, Nozzle2 and Nozzle-3. Each subfigure shows the profile achieved with high and low pulsation frequencies. The first remark lies in the acknowledgement that lower pulsation frequencies are associated systematically with faster swimming. This is further confirmed by plotting, in Fig. 3(d), the average speed for each of the runs performed.

The reason for the inverse correlation between speed and pulsation frequency is apparently related to the elastic nature of the thruster. In order for the robot to generate thrust, the chamber has to be properly refilled. This, however, depends both on the speed with which the elastic shell goes back to its unstrained state and the angular velocity of the rod. If the rod is revolving at a much faster rate than that required for the chamber to refill, the cables start pulling the shell before this has fully replenished the cavity. This, in turn, causes a

TABLE II

SUMMARY OF THE TESTS PERFORMED.

\begin{tabular}{|l|ccccc|}
\hline Test & pulsation $(\mathrm{Hz})$ & $\mathrm{L}^{\mathrm{D}} \mathrm{D}^{\mathrm{a}}$ & nozzle length & $\mathrm{D}(\mathrm{mm})$ & nozzle \\
\hline 1 & 1.88 & 4.7 & 20.0 & 30.0 & Nozzle-1 \\
2 & 2.66 & 4.7 & 20.0 & 30.0 & Nozzle-1 \\
3 & 1.88 & 4.7 & 60.0 & 30.0 & Nozzle-2 \\
4 & 2.66 & 4.7 & 60.0 & 30.0 & Nozzle-2 \\
5 & 1.88 & 16 & 60.0 & 20.0 & Nozzle-3 \\
6 & 2.66 & 16 & 60.0 & 20.0 & Nozzle-3 \\
\hline
\end{tabular}

${ }^{a}$ L/D is the thoeretical stroke ratio as defined by Gharib et al.(1998) [22], based on the maximum expelled volume. 
lower amount of fluid to be expelled, hence generating less thrust.

In addition, the vortical structures which issue from the nozzle play a significant role in improving the performance of the robot. The formation of a vortex ring at the nozzle-exit supports an excess thrust (e.g [18], [6], [19]). In order for this to occur, it is desirable that the ambient fluid enters across the ingestion valve and issues across the nozzle, as it happens in swimming cephalopods. If this is not the case and the refill occurs across the nozzle, vorticous fluid generated by the previous expulsion re-enters the nozzle and may hinder the formation of a new vortex ring, hence suppressing the benefit from nozzle-exit over-pressure (see [19]). Video recordings show that fast pulsation sequences hinder the proper working routine of the inflow valve, which causes the refill to take place across the nozzle and so depletes the generation of thrust. This explains why, with the current design, slow pulsations may be beneficial for the robot performances.

The occurrence of over-pressure due to vortex ring formation also explains why Nozzle-1 and Nozzle-2 outperform Nozzle-3 at low pulsation frequency. Nozzle-exit overpressure is associated with a characteristic non-dimensional formation time $T^{\prime}=\bar{q} t / d_{n}$, where $\bar{q}$ is the average outflow speed, $t$ the time during which flow has been ejecting and $d_{n}$ the nozzle-exit diameter. An optimal formation number exists for $T^{\prime} \approx 4$ at which excess thrust due to vortex ring generation is maximized (see Fig. 6 of Krueger and Gahrib [18]). If it is estimated that, at low frequency, a maximum volume of $100 \mathrm{ml}$ is actually ejected by the robot, the estimated formation times for Nozzle-1, Nozzle-2 and Nozzle- 3 are respectively $4.7,4.7$ and 16 . This suggests that, in the first two cases, the formation time is close to the optimal value, hence meeting the condition for the enhancement of thrust production. Nozzle-3 is well beyond the range where over-pressure can aid in the production of thrust and therefore its performances drop dramatically.

The prototype has served the purpose of demonstrating the feasibility of the cable-driven pulsating propulsion in a soft vehicle. The tests have provided us with evidence of a peculiar behaviour according to which an inverse correlation exists between speed and frequency of pulsation of the robot. Given the novelty of this kind of vehicle and mode of propulsion, there comes the need to derive a model to quantify the effects which the various design parameters play on the robot performances.

\section{Robot Model}

A simple model is devised according to the general design concepts illustrated in section 2 , which roughly relates the kinematics of the silicone shell deformation to the dynamics of the swimming robot. This model solves the standard onedimensional momentum equation for a neutrally buoyant, rigid body translating in water,

$$
M_{t o t} \frac{d U}{d t}=-\frac{1}{2} C_{d} U^{2} A_{r e f} \rho-X_{A M} \frac{d U}{d t}+q \frac{d m}{d t}
$$

where $U$ is the robot speed and $M_{t o t}=M+m$ is the overall mass of the robot comprising of $M$, the silicone and

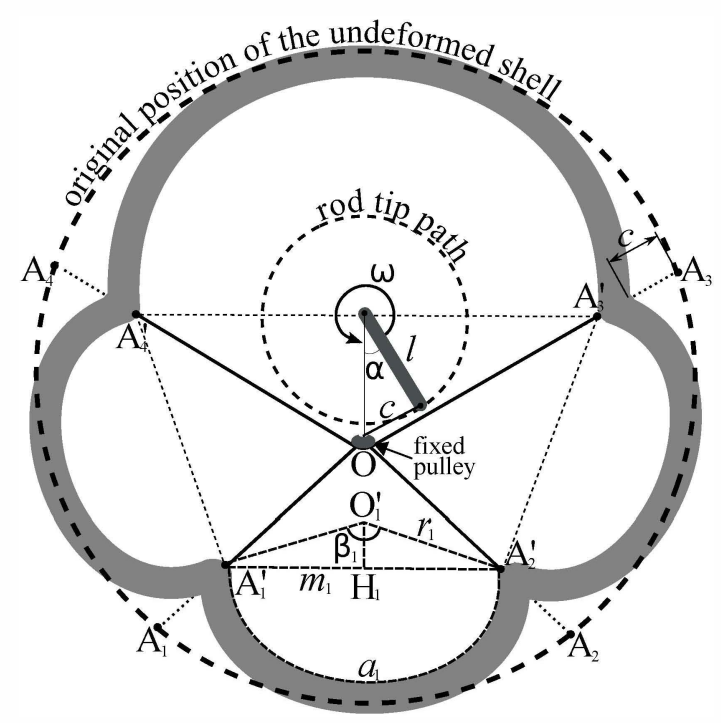

Fig. 4. Frontal view of a cross section of the robot. The outer dashed, bold line represents the unstrained elastic shell, while the light-grey, lobed strip represents the elastic shell subject to a contraction $c$ of the cables. Vertices $A_{i}$ and $A_{i}^{\prime}$ respectively represent the points of attachment of the cables to the shell prior to cable contraction. Vertex $O$ defines the position of the fixed pulley, while the dark-grey beam of length $l$ is the rod fitted onto the motor shaft.

mechanical/electrical parts and $m$, the mass of water stored in the chamber. The first right hand side (RHS) term represents the drag, $C_{d}$ being the drag coefficient, $A_{r e f}$ a reference area of the body and $\rho$ the fluid density; the second RHS term is the added mass, with $X_{A M}$ being the axial added mass coefficient, and the third term is the thrust, given by the speed of the outflow $q$ across the nozzle-exit area $A_{N}$ and the variation of mass $d m / d t$ occurring within the robot due to the cable-driven collapse of the elastic chamber. The thrust term is defined by the variation of mass which occurs inside the chamber due to localized cross sectional collapse of the elastic shell. The $d m / d t$ term can thus be expressed as a rate of change of the area within the cable affected cross section. By computing the displacement which the discrete points over the shell undergo due to cable shortening, the $d m / d t$ term in eq. (1) can be reformulated in terms of areal variation as follows,

$$
\frac{d m}{d t}=\rho \frac{d}{d t}\left(\Delta S A_{s}\right) .
$$

Here $A_{s}$ stands for the cross sectional area at time $t$, while $\Delta S$ defines a length scale of the region affected by the point-wise collapse of the elastic shell. $\Delta S$ physically represents the spatial extent of the shell along the axial direction which gets pulled radially inward by the cables. This term is dependent on the local value of the bending moment of the shell and, hence, on the degree of contraction exerted by the cables as well as on the thickness and elastic modulus of the silicone. However, for the present case, $\Delta S$ will be simply regarded as a somewhat empirical parameter linearly dependent on the contraction $c$ of the cables and on a coefficient $C_{s}$,

$$
\Delta S=C_{s} c .
$$


As far as the outflow speed is concerned, this is defined relative to the speed of the ambient flow, hence requiring a subtraction by the robot speed,

$$
q=C_{f} \frac{1}{\rho A_{N}} \frac{d m}{d t}-U
$$

where $C_{f}$ defines a flow loss coefficient at the nozzle entrance, which varies between 0.6 and 1 [23]. Substituting the term $d m / d t$ from eq. (2), eq. (4) becomes,

$$
q=\frac{C_{f}}{A_{N}}\left[\frac{d}{d t}\left(\Delta S A_{s}\right)\right]^{2}-U .
$$

The thrust term is thus rewritten,

$$
q \frac{d m}{d t}=\left\{\frac{C_{f}}{A_{N}}\left[\frac{d}{d t}\left(\Delta S A_{s}\right)\right]^{2}-U\right\}\left[\rho \frac{d}{d t}\left(\Delta S A_{s}\right)\right] .
$$

In order to solve eq. (6) a model is necessary which describes how the cross sectional area, $A_{s}$, pulled by the cables, varies in time.

The kinematic model for the silicone deformation is conceived as follows: the shaft of the gearmotor revolves at a prescribed rate $\omega$, this drives the rotation of the rod of length $l$ onto which $N$ cables are fitted, Fig 4 . The cables run through a fixed spot (i.e. the fairlead mentioned in section 2), vertex $O$ in Fig 4 and are linked to $N$ spots over one circumferential cross section of the elastic shell (vertices $A_{i}$ in Fig 4), which not necessarily need to be equispaced. Because all the cables are gathered through the fixed pulley, once the rod starts revolving around the shaft, they all get pulled the same length $c$, as depicted in Fig. 4. The extent by which the cables are pulled is related to the angle, $\alpha$, spanned over by the rod. Hence, given a timestep $d t$, the contraction $c$ is equivalent to the chord delimited by the tip of the rod at a certain instant and the position of the fixed pulley,

$$
c=2 l \sin \frac{\alpha}{2}
$$

where,

$$
\alpha=\int \omega d t
$$

being $0<\alpha<2 \pi$. This in turn provides us with the radial, inward displacement of the points of attachment of the cables onto the shell (i.e. vertices $A_{i}^{\prime}$ in Fig 4). The shortening of the cables deforms the shell into a lobe-shaped geometry with $i=N-1$ lobes, as depicted in Fig. 4. Focusing on one of the lobes (say lobe $i=1$ ), we see that points $A_{1}$ and $A_{2}$ in Fig. 4 are displaced to their new positions $A_{1}^{\prime}$ and $A_{2}^{\prime}$. The model employed here approximates these newly generated lobes to arcs of circumferences. The major limitation of this model is that contraction which cause highly distorted deformations of the shell, i.e. for which the approximation to an arc of circumference is not appropriate, cannot be accounted for. This poses a limitation to the extent of contraction which can be modelled. By approximating the lobes to arcs of circumference we can compute the reduction of cross sectional area in terms of the change from the initial circular sector $A_{1} \widehat{O} A_{2}$ to $A_{1}^{\prime} \widehat{O} A_{2}^{\prime}$. To do so, we define $a_{1}$,

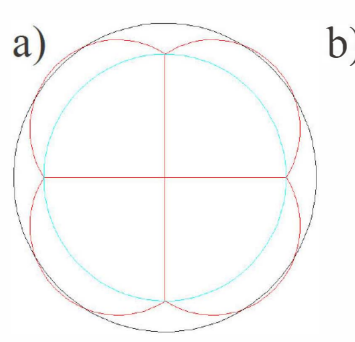

b)
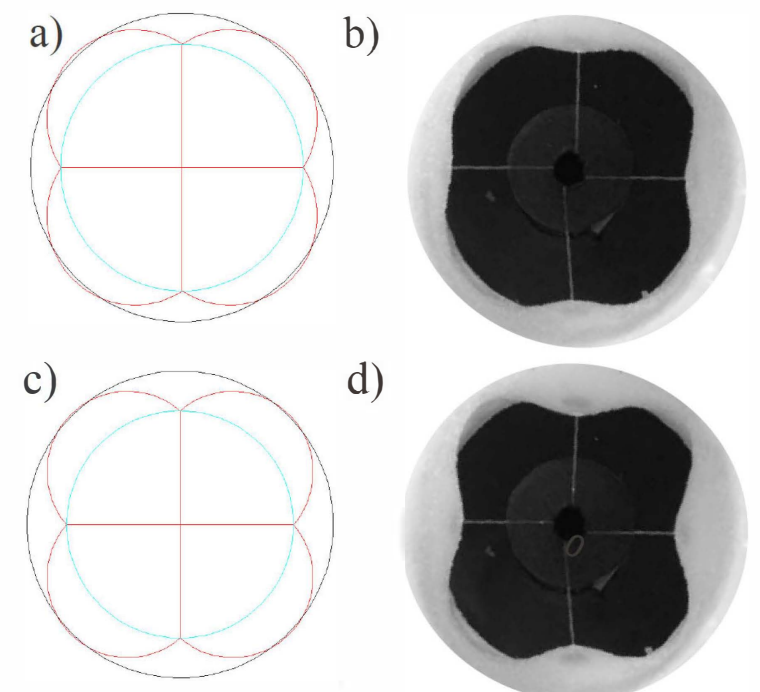

d)

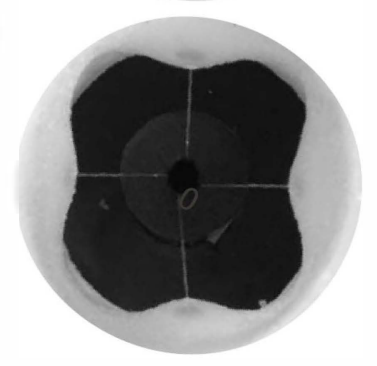

Fig. 5. Comparison between the simulated (a), (c) and observed (b), (d) deformation of the elastic shell.

$m_{1}, r_{1}$ and $\beta_{1}$ respectively as the arc, the chord, the radius and the angle subtended by $a_{1}$. Of all these, only $a_{1}$ and the respective coordinate positions of $A_{1}^{\prime}$ and $A_{2}^{\prime}$ (and therefore $\left.m_{1}\right)$ are known. Given $a_{1}$ and $m_{1}$, it is possible to compute the area of the circular sector $A_{1}^{\prime} \widehat{O} A_{2}^{\prime}$ by noticing that,

$$
\frac{\overline{H_{1} A_{2}^{\prime}}}{\overline{O_{1}^{\prime} A_{2}^{\prime}}}=\frac{m_{1}}{2 r_{1}}=\sin \frac{\beta_{1}}{2} \text {. }
$$

Since $r_{1}=\frac{a_{1}}{\beta_{1}}$, then,

$$
\frac{m_{1}}{2 a_{1}} \beta_{1}-\sin \frac{\beta_{1}}{2}=0
$$

The solution to this non-linear equation by Newton-Raphson iterative method provides us with a value for $\beta_{1}$, and therefore $r_{1}$. This enables us to compute the area Area $_{1}$ comprised between $m_{1}$ and $a_{1}$ by subtracting the area of the triangle $A_{1}^{\prime} \hat{O}_{1}^{\prime} A_{2}^{\prime}$ to that of the circle sector $\widehat{A_{1}^{\prime}} \widehat{O_{1}^{\prime}} A_{2}^{\prime}$ :

$$
\text { Area }_{1}=\frac{1}{2} r_{1} a_{1}-r_{1} \cos \left(\frac{\beta_{1}}{2}\right) \frac{m_{1}}{2} .
$$

By repeating this operation over each lobe, the crosssectional area $A_{s}$ associated with the contraction $c$, at time $t$, of the cables is obtained by:

$$
\begin{aligned}
A_{s}= & \sum_{i=1}^{N-1}\left[\frac{1}{2} r_{i} a_{i}-r_{i} \cos \left(\frac{\beta_{i}}{2}\right) \frac{m_{i}}{2}\right] \\
& +\frac{1}{2}\left(\sum_{i=1}^{N-2} x_{A_{i}^{\prime}} y_{A_{i+1}^{\prime}}+x_{A_{N-1}^{\prime}} y_{A_{1}^{\prime}}\right. \\
& \left.-\sum_{i=1}^{N-2} x_{A_{i+1}^{\prime}} y_{A_{i}^{\prime}}-x_{A_{1}^{\prime}} y_{A_{N-1}^{\prime}}\right),
\end{aligned}
$$

where the second RHS term is Gauss's formula for the area of the internal polygon defined by $A_{i}^{\prime}$ vertices and $\left(x_{A_{i}^{\prime}}, y_{A_{i}^{\prime}}\right)$ are the coordinates of vertex $A_{i}^{\prime}$. 
The kinematic model is tested with a 4-cable, equispaced configuration with the fixed pulley located at the centre of the cross section. Qualitative comparison of the results from the model with a silicone mock-up are provided in Fig. 5, where it is demonstrated that the geometrical model approximates the actual deformation of the silicone cylinder with a satisfactory degree of accuracy.

So far the model is capable of computing the area within a point-wise loaded circular shell, given the angle $\alpha$ of the rod. In order for this to be employed in the model of eq. 1 , a rate of change of the cross-sectional area is needed. Therefore a description of the temporal evolution of the area is required. In section IV the interplay between motor rotation and the resilience of the shell was shown to be a key element in determining the dynamics of the robot. Here we derive a simple formulation for such interaction.

Given a constant angular velocity of the motor, the temporal displacement of a point of attachment of the cable to the shell pulled by the rod is described by eq. 7, the solution of which is depicted in Fig. 6(a). Each rotation of the rod draws a trough-like profile of the displacement of the cable attachment. The deepest point of the trough corresponds to the maximum contraction, beyond which the rod starts to release the cable. The peak in the profile corresponds to the unstrained state of the shell. The case depicted in Fig. 6(a) represents the condition in which the point on the shell moves synchronously with the rod during both contraction and release of the cable, hence the profiles overlap. However, the time it takes the shell to go back to its unstrained state may be longer than the time it takes the rod to cover the second half-rotation, therefore, when the rod completes one full rotation the cables are still loose because the shell is still in the process of inflating. The contraction can start over only when the cables tighten, which occurs at the point of intersection between the profile drawn by the rod and that drawn by the shell in Fig. 6(b). The resilience of the shell is described here by a simple quadratic function in which a coefficient $V_{e}$ determines how fast the shell inflates.

The temporal profile of the contraction $c$ achieved by this latest addition to the kinematic model is employed to determine $A_{s}$. Eventually, by substituting $A_{s}$ from eq. (12) into eq. (6), eq. (1) can be easily solved numerically by the explicit Euler method.

\section{MODEL VALIDATION}

The model described in section 5 is employed to simulate the experiments with Nozzle-1 presented in section 3. Two runs are performed respectively setting $\omega=3.76$ and $\omega=$ $5.32 \mathrm{rad} / \mathrm{sec}, r=4, C_{d}=0.8$ and $C_{f}=0.6$. The added mass coefficient $X_{A M}$ is defined according to [24]. The empirical coefficient in the expression for $\Delta S$, eq. (3), is set to 3.0; this guarantees that the maximum ejected volume in the model is analogous to that in the experiments. The resilience of the silicone shell is expressed by means of the coefficient $V_{e}=9$ which sets the inflation time of the shell to $0.67 \mathrm{sec}$, close to the observed value in the experiments.
Results are presented in Fig. 7(a) for the low pulsation frequency case and Fig. 7(b) for the high pulsation frequency. In subfigure (a) and (b) of Fig. 7 the simulated results are compared respectively with the low-pass filtered recorded velocities of test 1 and test 2 of Table II. The simplified interplay between the shell and the motor accounted for in section $\mathrm{V}$ permits to capture the inverse correlation between pulsation frequency and speed of the robot. Indeed, having empirically defined a fixed speed of shell inflation, the faster the rod rotates, the less the cavity has managed to refill hence decreasing the thrust generated at each pulsation. The model describes the drop in performances from the low pulsation frequency to the high pulsation frequency, however, it appears that the complex physics associated with the unsteady mode of propulsion makes it non-trivial to output accurate quantitative estimates of the robot velocity profile. The low pulsation frequency test is well captured as far as the transient evolution to the steady state swimming regime is concerned. The model estimates lower long-term speed as those observed in the experiments. This can be attributed to the model not accounting for the vortex-enhanced thrust. In the test performed at high pulsation frequency, simulation and experiments only agree on the terminal velocity. The fact that the model does not describe the evolution from still to steady-state velocity seems to highlight the lack of the added mass cross terms in eq. 1. As a matter of fact, the fast jerking of the silicone shell at high pulsation frequency can definitely constitute a non-negligible effect on the dynamics of the robot during the early phase of acceleration. Of course, disturbances in the experiments due to wave generation and the occurrence of the float at the free surface, as well as the additional added mass effect due to the proximity of the lateral walls of the tank, are responsible for part of the mismatch between experiment and simulation. Most importantly, the physical model neglects the effect of fluid suction through the nozzle which occurs during the refill
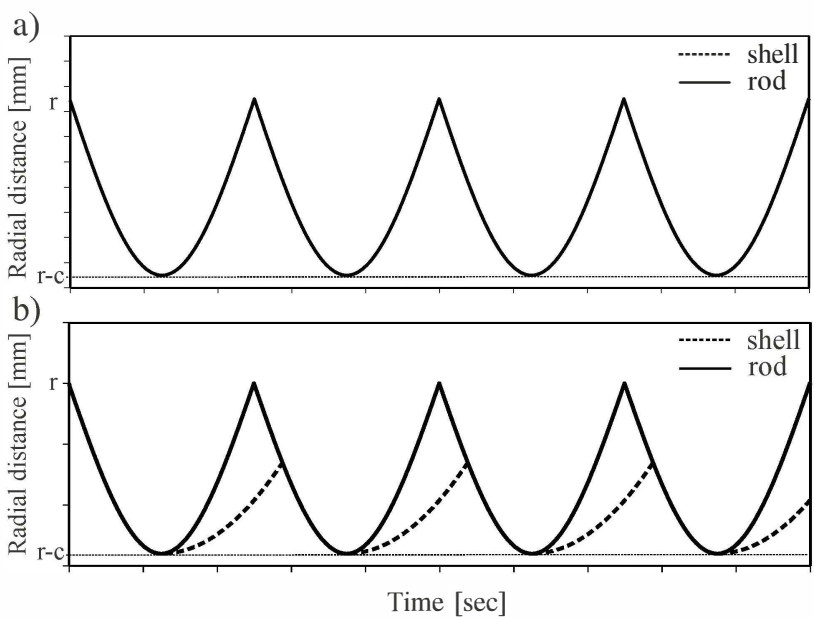

Fig. 6. Temporal profile of a point of attachment of the cable to the silicone shell in (a), the case where the shell inflates synchronously with the release of the cables and, (b) the case where the inflation time of the shell is slower than the release of the cables by the rod. 

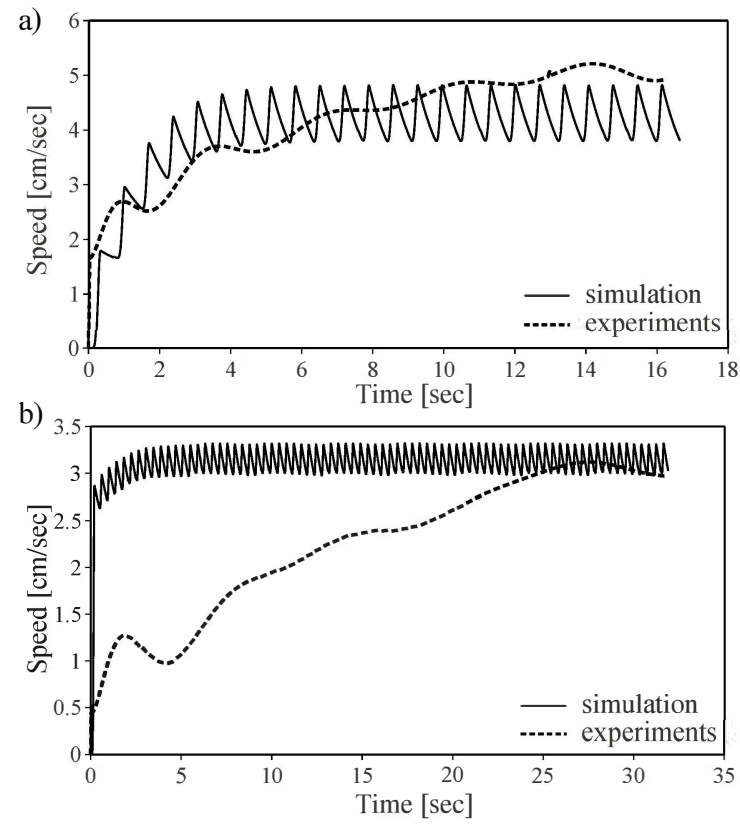

Fig. 7. Comparison of the robot speed as achieved by the simulation and from recordings of test-1 (a) and test-2 (b) of Table II.

phase. Because at high pulsation frequency the pressure drop in the chamber is not sufficient to make the nozzle fully collapse and, in this way, impede the backflow across the siphon, ambient fluid must be sucked into the cavity causing the onset of a negative contribution to the thrust term. In order to better account for this effect, a closer investigation of the fluid-structure interaction along the nozzle length is required.

\section{CONCLUSION}

In this paper a new kind of underwater robot is presented. This robot is innovative both in its design, being for the most part soft, and in the way it propels itself, that is by a pulsedjet swimming routine. The robot swims by sequentially collapsing an external elastic shell and then refilling it with ambient water. The contraction of this chamber is actuated by cables radially pulled by a gearmotor, while the expansion of the chamber spontaneously occurs due to the stresses generated within its thickness by the strain occurred during the contraction. The collapse of the shell accelerates fluid across a nozzle which, by reaction, generates thrust.

A first series of test is performed, the major outcome of which is the acknowledgement of an inverse correlation between pulsation frequency and speed of the robot. This is due to the limitation of the elastic shell to inflate fast enough. Indeed, if the chamber is not refilled, the subsequent contraction cannot expel a volume of fluid sufficient to generate the thrust needed to propel the robot. This in part explains why slower pulsation routines perform better. On the other hand, observations of the opening/closing sequence of the ingestion valve and the nozzle suggest that better performances could be associated with the formation of ordered vortex rings which support the onset of additional thrust. A model is also developed to try and reproduce the dynamics of the robot in terms of the kinematics of the elastic shell. This model describes the interplay between the contraction of the shell driven by a revolving rod and the inflation of the shell due to the elastic nature of its constituent material. From the description of these two terms the deformation of the external shell is derived and, from these, geometrical arguments are employed to compute the area within the collapsing cross section of the shell. The area computed in this way is eventually fed into the thrust term in the equation for the momentum of the robot. The model is tested in two scenarios with low and high pulsation frequency demonstrating its fitness at capturing the key elements of the pulsed propulsion.

This work represents a first insight into what could be referred to as marine soft robotics by presenting a new self-propelling, soft vehicle and attempting to describe the complex dynamics associated with its unconventional mode of propulsion. These first set of observations disclose a whole new range of problems which need to be accounted for. Future development will address three major aspects of SUUVs design. First, an improved physical model will be devised which better accounts for the dynamics associated with the interplay between the collapsible nozzle and the ingestion valve. In addition, the authors acknowledge that the current performances of the vehicle are well below those observed in common cephalopods, this being due to the limited amount and speed of the expelled fluid. Therefore, in order to achieve a substantial improvement in the jetting performance of the thruster, larger volumes of expelled fluid are needed. To this end the current actuator will be revisited in order to enable the contraction of cables distributed over the entire surface of the vehicle cavity as well as a more impulsive collapse of the cavity. Finally, steering of the vehicle will be accounted for via the implementation of a tendon-driven, collapsible, thrust-vectoring nozzle which will provide the vehicle with a high degree of manoeuvrability without compromising the softness of the overall structure.

\section{REFERENCES}

[1] C. Zheng, S. Shatara, and T. Xiaobo, "Modeling of biomimetic robotic fish propelled by an ionic polymermetal composite caudal fin," IEEE Transactions on Mechatronics, vol. 15, pp. 448-459, 2010.

[2] J. Yu, R. Ding, Q. Yang, M. Tan, W. Wang, and J. Zhang, "On a bio-inspired amphibious robot capable of multimodal motion," IEEE Transactions on Mechatronics, vol. PP, pp. 1-10, 2011.

[3] J. E. Colgate and K. M. Lynch, "Mechanics and control of swimming: a review," IEEE Journal of Oceanic Engineering, vol. 29, pp. 660-673, 2004.

[4] L. A. Ruiz, R. W. Whittlesey, and J. O. Dabiri, "Vortex-enhanced propulsion," Journal of Fluid Mechanics, vol. 668, pp. 5-32, 2011.

[5] K. Mohseni, "Zero-mass pulsatile jets for unmanned underwater vehicle maneuvering," in AIAA 3rd 'Unmanned Unlimited' Techical Conference, Workshop and Exhibit, Chicago, IL, 2004.

[6] M. Krieg and K. Mohseni, "Thrust characterization of a bio-inspired vortex ring generator for locomotion of underwater robots," IEEE Journal of Ocean Engineering, vol. 33, 2008.

[7] A. A. Moslemi and P. S. Krueger, "Propulsive efficiency of a biomorphic pulsed-jet underwater vehicle," Bioinspiration and Biomimetics, vol. 5, pp. 1-14, 2010. 
[8] M. Krieg, P. Klein, R. Hodgkinson, and K. Mohseni, "A hybrid class underwater vehicle: bioinspired propulsion, embedded system, and acoustic communication and localization system," Marine Technology Society Journal, vol. 45, pp. 153-164, 2011.

[9] D. Trivedi, C. D. Rahn, W. M. Kier, and I. D. Walker, "Soft robotics: Biological inspiration, state of the art, and future research," Applied Bionics and Biomechanics, vol. 5, pp. 99-117, 2008.

[10] S. A. Morin, R. F. Shepherd, S. W. Kwok, A. A. Stokes, A. Nemiroski, and G. M. Whitesides, "Camouflage and display for soft machines," Science, vol. 337, no. 6096, pp. 828-832, 2012.

[11] C. Onal and D. Rus, "A modular approach to soft robots," in Biomedical Robotics and Biomechatronics (BioRob), 2012 4th IEEE RAS EMBS International Conference on, june 2012, pp. $1038-1045$.

[12] J. Hiller and H. Lipson, "Automatic design and manufacture of soft robots," Robotics, IEEE Transactions on, vol. 28, no. 2, pp. 457 -466, april 2012.

[13] M. Nokata and T. Sato, "Deformable robot maneuvered by magnetic particles for use in a confined environment," in Robotics and Automation (ICRA), 2012 IEEE International Conference on, may 2012, pp. $3739-3744$.

[14] A. Boxerbaum, A. Horchler, K. Shaw, H. Chiel, and R. Quinn, "Worms, waves and robots," in Robotics and Automation (ICRA), 2012 IEEE International Conference on, may 2012, pp. 3537 -3538.

[15] C. Laschi, B. Mazzolai, V. Mattoli, M. Cianchetti, and P. Dario, "Design of a biomimetic robotic octopus arm," Bioinspiration and Biomimetics, vol. 4, pp. 1-8, 2009.

[16] M. Cianchetti, A. Arienti, M. Follador, B. Mazzolai, P. Dario, and C. Laschi, "Design concept and validation of a robotic arm inspired by the octopus," Materials Science and Engineering C, vol. 31, pp. 1230-1239, 2011.

[17] M. Calisti, M. Giorelli, G. Levy, B. Mazzolai, B. Hochner, C. Laschi, and P. Dario, "An octopus-bioinspired solution to movement and manipulation forsoft robots," Bioinspiration and Biomimetics, vol. 6, pp. 1-10, 2011.

[18] P. S. Krueger and M. Gharib, "The significance of vortex ring formation to the impulse and thrust of starting jet," Physics of Fluid, vol. 15, pp. 1271-1281, 2003.

[19] P. S. Krueger, "An over-pressure correction to the slug model for vortex ring circulation," Journal of Fluid Mechanics, vol. 545, pp. 427-443, 2005.

[20] J. M. Gosline and M. E. DeMont, "Jet-propelled swimming squids," Scientific American, vol. 252, pp. 96-103, 1985.

[21] F. Giorgio Serchi, A. Arienti, I. Baldoli, and C. Laschi, "Biomimetic vortex propulsion: toward the new paradigm of soft unamnned underwater vehicles," IEEE/ASME Transaction on Mechatronics, vol. 18, pp. 484-493, 2013.

[22] M. Gharib, E. Rambod, and K. Shariff, "A universal time scale for vortex ring formation," Journal of Fluid Mechanics, vol. 360, pp. 121140, 1998.

[23] W. Johnson, P. D. Soden, and E. R. Trueman, "A study in jet propulsion: an analysis of the motion of the squid, Loligo Vulgaris," Journal of Experimental Biology, vol. 56, pp. 155-165, 1972.

[24] T. L. Daniel, "Cost of locomotion: unsteady medusan swimming," Journal of Experimental Bilogy, vol. 119, pp. 149-164, 1985. 